Polymer Journal, Vol. 8, No. 1, pp 21-29 (1976)

\title{
Diffusion in the Nylon 12 and Water System
}

\author{
Tadahiro Asada, Kazuo Inoue, ${ }^{*}$ and Shigeharu Onogi \\ Department of Polymer Chemistry, Kyoto University, Kyoto 606, Japan. \\ (Received April 1, 1975)
}

\begin{abstract}
Diffusion in a nylon 12 and water system has been measured by means of a quartz microblance and weighing bottle method at penetrant concentrations ranging from about 0.2 to $2(\mathrm{wt} \%)$ and temperatures ranging from 20 to $70^{\circ} \mathrm{C}$. The average diffusion coefficient $\bar{D}_{\mathrm{av}}$ for quenched nylon $12 \mathrm{film}$ and the apparent average diffusion coefficient $\bar{D}_{\mathrm{av}}^{*}$ for annealed nylon $12 \mathrm{film}$ as a function of penetrant concentration have been determined from the absorption-desorption data. The integral diffusion coefficient $\bar{D}_{\mathrm{s}}$ as a function of penetrant concentration has been determined from the steady-state behavior and compared with $\bar{D}_{\mathrm{av}}$ or $\bar{D}_{\mathrm{av}}^{*}$. The concentration dependences of $\bar{D}_{\mathrm{av}}$ and $\bar{D}_{\mathrm{av}}^{*}$ are somewhat different from each other, but the concentration dependences of $\bar{D}_{\mathrm{s}}$ for quenched film are the same as those for annealed film when plotted against the weight of water absorbed per unit weight of the amorphous part of the dry polymer. The solubility $S$ and the permeability $P$ in the nylon $12+$ water system are proportional to the volume fraction $X_{\mathrm{a}}$ of the amorphous phase. The mutual diffusion coefficient at the limit of zero penetrant concentration for this system is compared with those for other polymer + water systems.

KEY WORDS Nylon 12 / Water / Diffusion / Sorption / Absorption / Desorption / Permeability / Crystallinity /
\end{abstract}

In polymer and water systems, absorption and desorption processes are not always simple Fickian in nature. ${ }^{1}$ The water molecule is relatively small and is strongly associated by hydrogen bond formation in both the liquid and solid states. This combination of features distinguishes it from the majority of organic penetrants. Due to these properties of the water molecule, absorption and desorption processes in a hydrophilic polymer+water system are very complicated. ${ }^{1,2}$ Moreover, hydrophilic polymers in the solid state usually consist of crystalline and amorphous phases, which make the processes much more complicated. Among such hydrophilic crystalline polymer+water systems the diffusion in the nylon $6+$ water system has already been studied. ${ }^{2}$ In this system, the sorption process is essentially non-Fickian, though this is not evident in the absorption-desorption curves. In addition, the diffusion coefficient for this system depends not only on concentration but also on time or history, whereas the diffusion coefficients for hydrophobic polymer+water

* On leave from, Hirakata Plastic Laboratory, Ube Industries, Japan. systems are usually independent of water concentration and time. ${ }^{3-8}$

In this study, the nylon $12+$ water system has been chosen as a typical example of a less hydrophilic polymer+water system; sorption characteristics of nylon 12 are intermediate between those for hydrophilic and hydrophobic materials. The effect of crystallinity on the diffusion and sorption processes has also been investigated.

\section{EXPERIMENTAL}

\section{Materials}

Nylon 12 was prepared by condensation polymerization of dodecalactam at $300^{\circ} \mathrm{C}$ in the presence of a small amount of water. The crude polymer product obtained was purified by washing it several times with methanol to remove low-molecular-weight substances.

Quenched and annealed films of nylon 12 were employed in this study. The quenched film was prepared by extrusion of the above nylon 12 from a slot-die followed by cooling on a chilled roller. The thickness of the film was about $50 \mu$. The film thus prepared was washed with 
methanol-water mixture, and then dried in air. To prepare the annealed film, the quenched film was heated at $150^{\circ} \mathrm{C}$ in vacuo for $5 \mathrm{hr}$ and slowly cooled to room temperature. The film was then left in the room atmosphere for more than three months and dried over $\mathrm{P}_{2} \mathrm{O}_{5}$ in a desiccator for more than two weeks before measurements.

\section{Measurements}

The density of the films was measured by a flotation method using a toluene-carbon tetrachloride mixture at $30^{\circ} \mathrm{C}$. The densities for the quenched film and annealed film were 1.016 and $1.023 \mathrm{~g} / \mathrm{cm}^{3}$, respectively.

The density for the perfect crystal in the $\gamma$ form of nylon 12 is $1.045\left(\mathrm{~g} / \mathrm{cm}^{3}\right)^{9}$ and that in the perfectly amorphous state is $0.990\left(\mathrm{~g} / \mathrm{cm}^{3}\right)$; this was estimated by measuring the heat of fusion as a function of specific volume for various samples and extrapolating the heat of fusion to zero as shown in Figure 1. The volume percent crystallinity was calculated by the following equation from the densities,

$$
X_{\mathrm{c}}=\frac{\rho-\rho_{\mathrm{a}}}{\rho_{\mathrm{c}}-\rho_{\mathrm{a}}} \times 100
$$

where $\rho, \rho_{\mathrm{c}}$, and $\rho_{\mathrm{a}}$ are the densities of the sample film, the perfectly crystalline, and amorphous phases respectively.

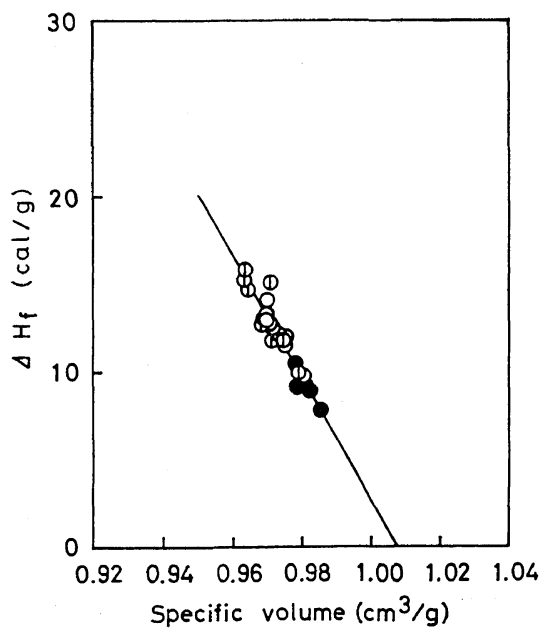

Figure 1. The heat of fusion $\Delta H_{\mathrm{f}}$ plotted against specific volume for various kinds of nylon 12 films. The different symbols in the figure show the different kinds of films obtained under different processing conditions.
The sorption apparatus employed in this study was essentially the same as the quartz microbalance apparatus described by Prager and Long. ${ }^{10}$ The integral absorption from and the integral desorption to zero pressure were measured at several external pressures of water vapor at several temperatures between 20 and $70^{\circ} \mathrm{C}$. In each measurement, the temperature of the system was controlled within $\pm 0.1^{\circ} \mathrm{C}$. The pressure of vapor in the sorption tube was measured with a mercury manometer to within $\pm 0.5 \mathrm{~mm}$.

The steady-state transmission of water vapor through the film was determined by a modification of the well-known cup method. The difference in our procedure was the use of two cups; inside the usual metallic cup having a diameter of $3 \mathrm{~cm}$, another cup made of glass was inserted to hold a proper salt solution for the purpose of humidity control. The cups equipped with the film sample and salt solution were placed over $\mathrm{P}_{2} \mathrm{O}_{5}$ in a desiccator, and the latter was kept in a water bath controlled at a constant temperature within $\pm 0.5^{\circ} \mathrm{C}$.

The transmission of water vapor was determined from a decrease in the weight of the cups measured after appropriate time intervals, which depended mainly upon the measuring temperature and were determined empirically. The weighing of the cups was carried out as rapidly as possible in order to minimize experimental errors. Three successive measurements were carried out for the same sample, and the results agreed always within $\pm 5 \%$. A mean value of them was taken as the transmission at a given humidity and temperature.

\section{RESULTS AND DISCUSSION}

\section{Absorption and Desorption}

Figure 2 shows an example of a reduced absorption-desorption curve for nylon $12+$ water system at $30^{\circ} \mathrm{C}$. In Figure 2 , the fractional uptake and loss of water $\left(M_{t} / M_{\infty}\right)$ are plotted against $\sqrt{t} / l$, where $M_{t}$ is the amount of the penetrant absorbed or desorbed in time $t, M_{\infty}$ the equilibrium penetrant concentration and $l$ the thickness of the sample. The absorption-desorption curves at constant temperature do not depend strongly on the vapour pressure 
Diffusion in the Nylon 12 and Water System

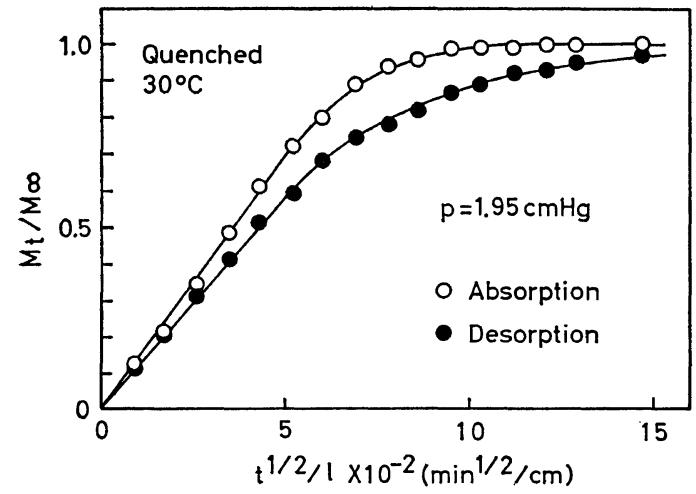

Figure 2. Absorption and desorption curves for the quenched film at $30^{\circ} \mathrm{C}$.

of the water but do depend greatly on the measuring temperature. Figure 3 shows an example of variation of the absorption-desorption curves with temperature for the quenched film at a relative humidity $p / p_{0}=0.60$. It is evident from this figure that the rate of absorption and desorption increases with increasing tem- perature.

The absorption curves for this system show that the diffusion process seems to be Fickian, because the absorption curves are straight up to $60 \%$ of the maxima and approach the maxima asymptotically without any inflection point. ${ }^{11}$ The desorption curves are always below the corresponding absorption curves, suggesting that the diffusion coefficient for this system depends on the penetrant concentration and increases with increasing penetrant concentration.

According to the current theories of diffusion ${ }^{1,11}$ the mutual diffusion coefficient $D$ can be obtained from the initial slope of the absorption and desorption curves when $D$ depends on concentration only. The initial diffusion coefficients $D_{\mathrm{a}}$ and $D_{\mathrm{d}}$ for absorption and desorption can be obtained by the following equations;

$$
D_{\mathrm{a}}=\pi I_{\mathrm{a}}{ }^{2} / 16, \quad D_{\mathrm{d}}=\pi I_{\mathrm{d}}{ }^{2} / 16
$$

where $I_{\mathrm{a}}$ and $I_{\mathrm{d}}$ are respectively the initial slopes of the reduced absorption and desorption curves. When the concentration dependence of $D$ is

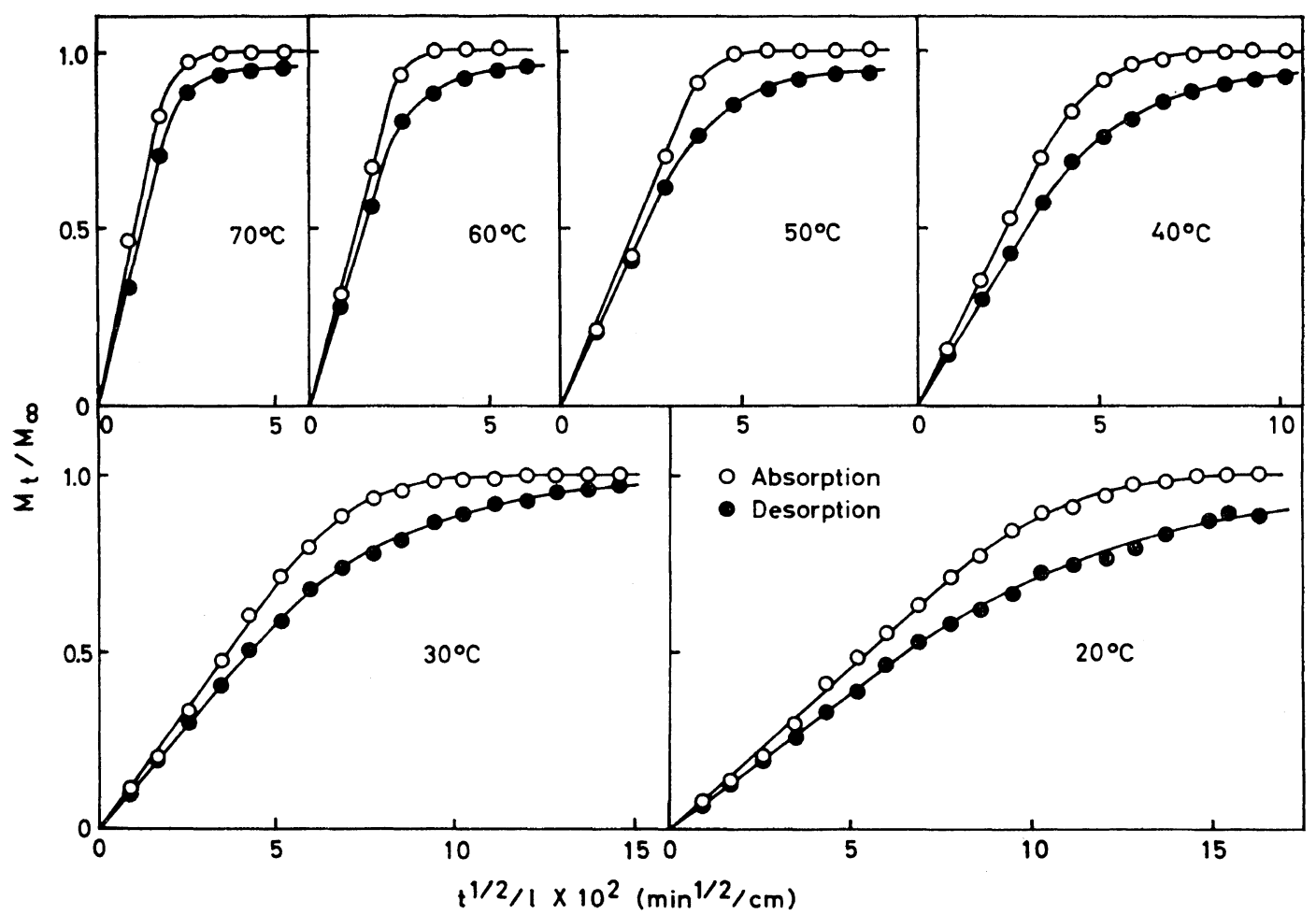

Figure 3. Absorption and desorption curves for the quenched film at various temperatures at a relative humidity $p / p_{0}=0.60$. 


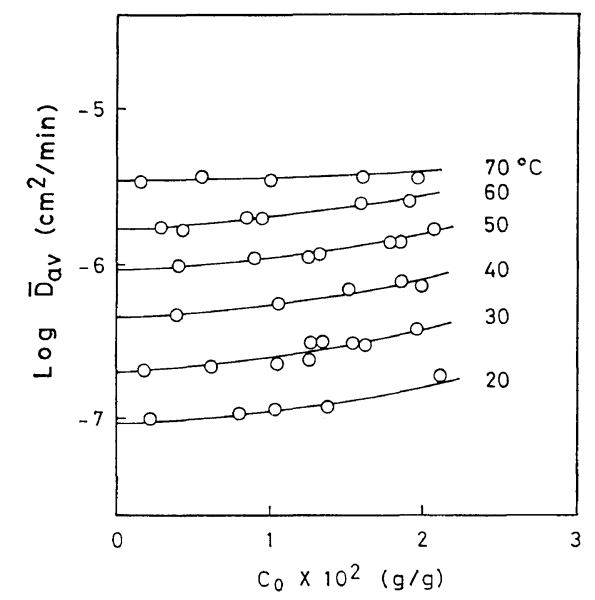

Figure 4. The average diffusion coefficient $\bar{D}_{\mathrm{av}}$ vs. equilibrium penetrant concentration for the quenched nylon 12 film + water system at various temperatures.

small, the integral diffusion coefficient $\bar{D}$ is given approximately by the following equation;

$$
\bar{D}=\frac{1}{c_{0}} \int_{0}^{c_{0}} D \mathrm{~d} c \fallingdotseq \bar{D}_{\mathrm{av}}=\frac{D_{\mathrm{a}}+D_{\mathrm{d}}}{2}
$$

where $c, c_{0}$, and $\bar{D}_{\mathrm{av}}$ are the penetrant concentration, the equilibrium penetrant concentration, and the average diffusion coefficient, respectively. $\bar{D}_{\mathrm{av}}$, evaluated from eq 1 and 2 for the quenched film at various temperatures and pressures, is plotted against the equilibrium penetrant concentration $c_{0}$ in Figure 4. The value of $\bar{D}_{\mathrm{av}}$ for this system is smaller than that for the nylon $6+$ water system under the same condition. As seen from Figure $4, \bar{D}_{\text {av }}$ depends slightly on the water concentration below $60^{\circ} \mathrm{C}$ and becomes constant at $70^{\circ} \mathrm{C}$. Since the glass transition temperature $T_{\mathrm{g}}$ of the nylon 12 is estimated to be about $40^{\circ} \mathrm{C},{ }^{12}$ it can be said that $\bar{D}_{\mathrm{av}}$ becomes independent of the penetrant concentration at temperatures well above $T_{\mathrm{g}}$ and depends on penetrant concentration near and below $T_{\mathrm{g}}$.

\section{Steady-State Behavior}

As pointed out in the previous paper, ${ }^{2}$ for the purpose of judging whether the system to be Fickian or not, it is most useful to compare the $D$ value obtained by a transient method (such as the absorption-desorption method) with that from a steady state method. The mutual diffusion coefficient which depends on concen-

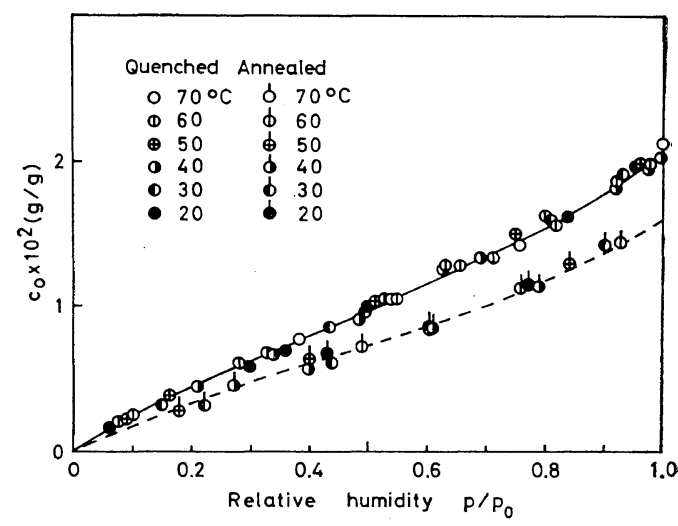

Figure 5. The equilibrium penetrant concentrations for the quenched and annealed samples at various humidities and temperatures.

tration can be obtained by the steady-state permeability method, even if the diffusion coefficient depends on time.

As mentioned above, the equilibrium penetrant concentration $c_{0}$ was determined using a quartz helix microbalance apparatus. The equilibrium penetrant concentration determined by this method is plotted against the relative humidity, $p / p_{0}$, in Figure 5. As is evident from this figure, the equilibrium sorption isotherm is independent

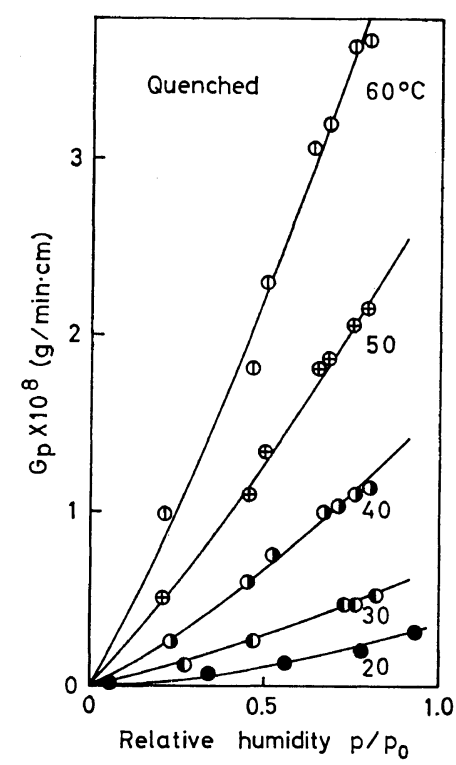

Figure 6. The steady-state rate of transmission $G_{p}$ vs. relative humidity for the quenched sample at various temperatures. 
of temperature. From the slope of the transmission vs. time curve in the steady state, the steady-state rate of transfer $F$ and hence the product $F \cdot l=G_{p}$, can be evaluated. $G_{p}$ thus determined for the quenched samples at various temperatures is plotted against the relative humidity in Figure 6.

The $G_{p}$ is related to the integral diffusion coefficient $\bar{D}_{\mathrm{s}}$ by the equation,

$$
\bar{D}_{\mathrm{s}}=\frac{G_{p}}{c_{0}}
$$

where $c_{0}$ is the penetrant concentration in $\mathrm{g} / \mathrm{cm}^{3}$. $\bar{D}_{\text {s }}$ denotes the integral diffusion coefficient evaluated from the data obtained by the steadystate permeation measurements. Here, to evaluate $\bar{D}_{\mathrm{s}}$, the calculated value from the $c_{0}$ in $\mathrm{g} / \mathrm{g}$ was used for $c_{0}$ in $\mathrm{g} / \mathrm{cm}^{3}$, assuming the additivity of volume of the polymer in the dry state and that of the penetrant. The integral diffusion coefficient $\bar{D}_{\mathrm{s}}$ evaluated from the $G_{p}$ and $c_{0}$ at various temperatures is plotted against the penetrant concentration $c_{0}$ as the closed circles in Figure 7 , and is compared with the average diffusion coefficient $\bar{D}_{\mathrm{av}}$ (open circles) obtained by the absorption-desorption method. As seen from this figure, the two diffusion coefficients do not deviate much from each other at any temperature, suggesting that the sorption process of this system is approximately Fickian. The slight deviation between the concentration dependences of the two diffusion coefficients

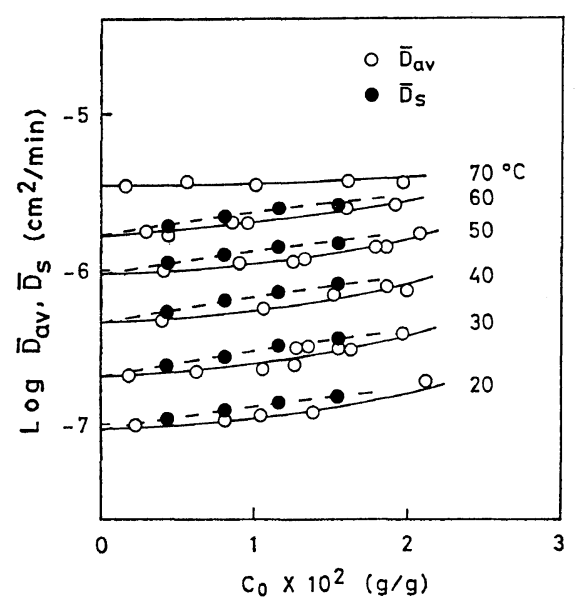

Figure 7. A comparison of $\bar{D}_{\mathrm{S}}$ with $\bar{D}_{\mathrm{av}}$ for quenched nylon 12 film + water system. implies that the sorption process has a slight non-Fickian character, which appeared more obviously in the nylon $6+$ water system reported previously. $^{2}$

\section{Effect of Annealing}

Figure 8 shows an example of the absorptiondesorption curves for the annealed film of nylon 12 at $30^{\circ} \mathrm{C}$. As seen in this figure the initial part of both the absorption and desorption curves for the annealed film is not linear and curves slightly downward, indicating that the diffusion is no longer Fickian. Although the figures are not shown here, the initial part of the absorption-desorption curves for the annealed films at higher temperatures is linear. The true diffusion coefficient for non-Fickian systems cannot be evaluated from the initial slope of the

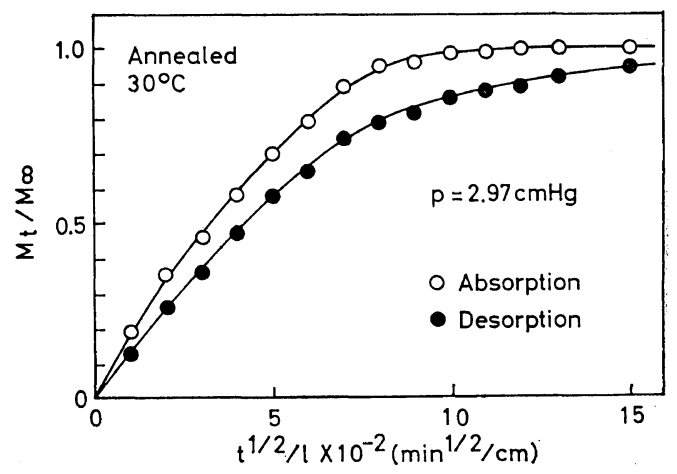

Figure 8. Absorption-desorption curves for the annealed film at $30^{\circ} \mathrm{C}$.

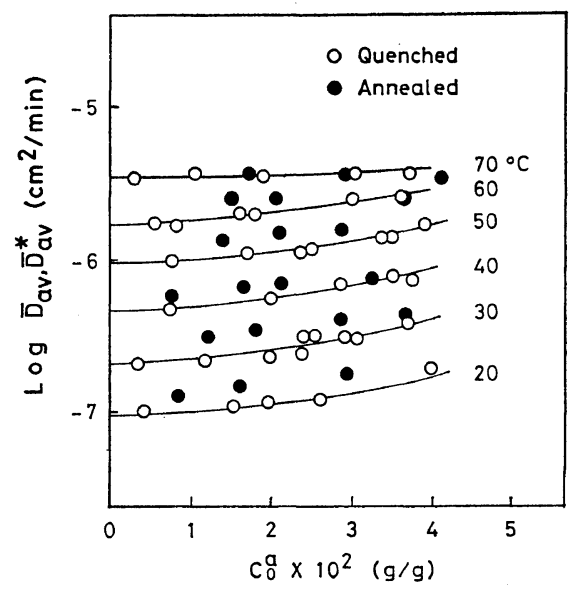

Figure 9. A comparison of $\bar{D}_{\mathrm{av}}^{*}$ with $\bar{D}_{\mathrm{av}}$. 
absorption-desorption curves, but an apparent average diffusion coefficient $\bar{D}_{\mathrm{av}}^{*}$ was evaluated by trial from the initial slope of the absorptiondesorption curves in Figure 8 and compared with the result for the quenched film in Figure 9. In Figure $9, \bar{D}_{\mathrm{av}}^{*}$ is plotted against the water content of the amorphous phase in the polymer, $c_{0}{ }^{a}(\mathrm{~g} / \mathrm{g})$, and compared with $\bar{D}_{\mathrm{av}}$ for the quenched sample. At temperatures higher than $60^{\circ} \mathrm{C}$ both $\bar{D}_{\mathrm{av}}^{*}$ and $\bar{D}_{\mathrm{av}}$ coincide well with each other, while at temperatures lower than $50^{\circ} \mathrm{C}$ they deviate from each other. The increase in crystallinity by annealing seems to change the diffusion characteristics from slightly non-Fickian to completely non-Fickian. The equilibrium sorption isotherm for the annealed sample shown in Figure 5 differs from that for the quenched one. The regain of water is reduced by annealing at any relative humidity $p / p_{0}$. Since the crystal modification of both samples was ascertained to be the same ( $\gamma$-form) by X-ray diffraction measurements, ${ }^{9}$ the difference in the water regain between these two samples is probably caused by differences in crystallinity. In Figure 10, the equilibrium water regain $c_{0}$ is plotted against volume fraction $X_{\mathrm{a}}$ of the amorphous phase at various humidities. As seen from this figure, the equilibrium regain is proportional to the volume fraction of the amorphous phase in the polymer.

Figure 11 shows the steady-state rate of transfer $G_{p}$ for the annealed sample plotted against relative humidity $p / p_{0}$. As is evident from this figure,

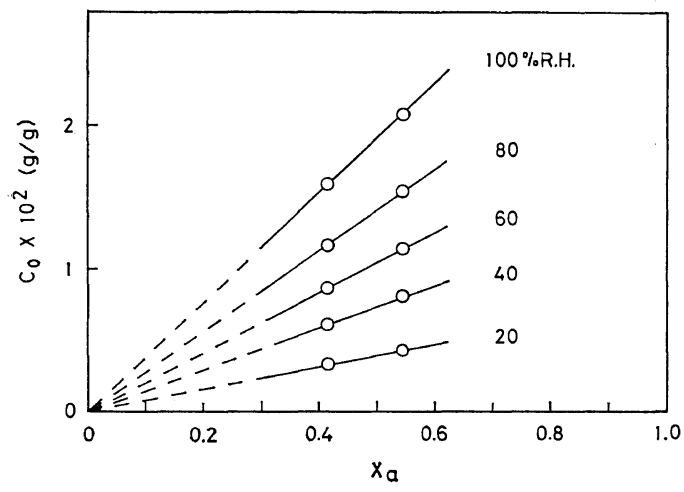

Figure 10. The equilibrium penetrant concentration vs. amorphous volume fraction of the polymer at various humidities.

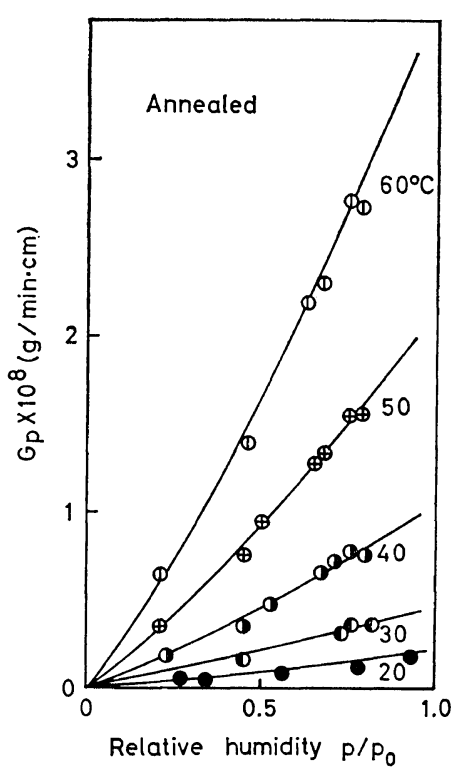

Figure 11. The steady-state rate of transmission $G_{p}$ vs. relative humidity for the annealed film at different temperatures.

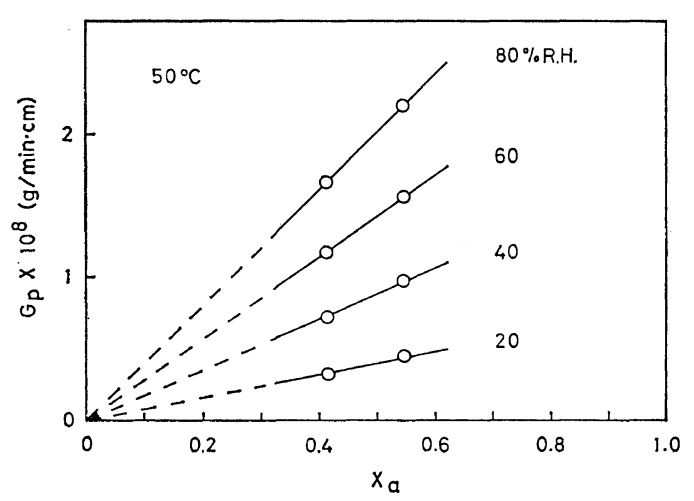

Figure 12. $G_{p}$ vs. $X_{\mathrm{a}}$, amorphous volume fraction of the polymer, at various humidities.

$G_{p}$ is also reduced by annealing. In Figure 12, the steady-state rate of transfer $G_{p}$ is plotted against the volume fraction of the amorphous phase. As seen from this figure, $G_{p}$ is also proportional to the volume fraction of the amorphous phase. The integral diffusion coefficient $\bar{D}_{\mathrm{s}}$ determined from $G_{p}$ and $c_{0}$ for the annealed sample is plotted against $c_{0}{ }^{a}$ and compared with that for the quenched one in Figure 13. The integral diffusion coefficients $\bar{D}_{\mathrm{s}}$ for both the samples agree well with each other, being con- 


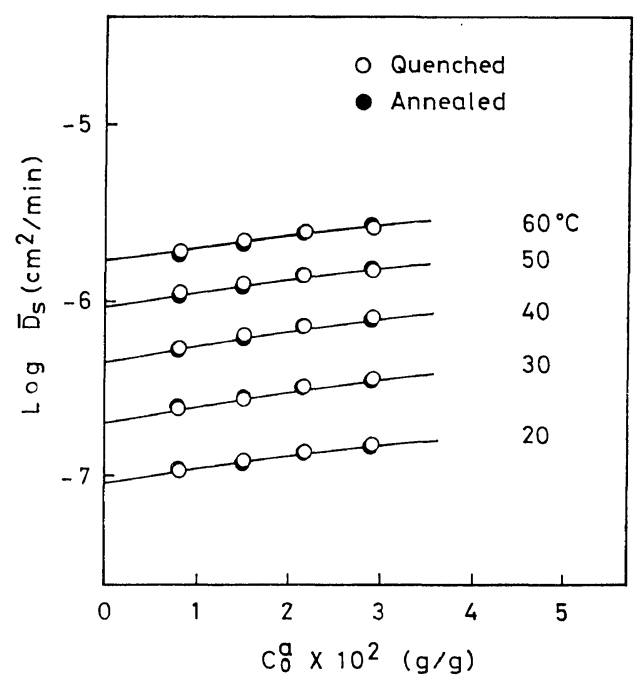

Figure 13. Integral diffusion coefficient $\bar{D}_{\mathrm{s}}$ for nylon 12 +water system against penetrant concentration $c_{0}{ }^{a} . \quad c_{0}{ }^{a}$ is defined as the weight of water absorbed per unit weight of the amorphous part of the dry polymer.

trary to the result for the average diffusion coefficient $\left(\bar{D}_{\mathrm{av}}\right.$ and $\left.\bar{D}_{\mathrm{av}}^{*}\right)$ obtained from the absorption-desorption method shown in Figure 9.

The fact that $\bar{D}_{\mathrm{s}}$ for both the samples agrees well with each other implies that the mutual diffusion coefficient $D$ in the steady state for both the samples also agrees (see Figure 14), indicating that the mutual diffusion coefficient $D$ in the steady state is not affected by annealing. On the contrary, the fact that the average diffusion coefficients for the samples obtained from the absorption-desorption method does not agree with each other (as shown in Figure 9) is considered to be due to the time dependence of the mutual diffusion coefficient being affected by annealing.

Now let us assume that the diffusion coefficient $D$ for a time-dependent diffusion can be expressed by following equation proposed by Crank. ${ }^{13}$

$$
\frac{\partial D}{\partial t}=\frac{\partial D_{\mathrm{i}}}{\partial t}+\alpha\left(D_{\mathrm{e}}-D\right)
$$

Here $D_{\mathrm{i}}$, that part of $D$ which can change instantaneously, is a function of concentration only but is not influenced by changes in any other variable. $D_{\mathrm{e}}$ is the equilibrium diffusion coefficient and $\alpha$ is the rate parameter controlling the approach to equilibrium. The difference between $\bar{D}_{\mathrm{av}}$ and $\bar{D}_{\mathrm{av}}^{*}$ may be caused by a difference in $\alpha$ for each sample. For instance, $\alpha$ for the quenched film is considered to be smaller than that for the annealed one. The true mutual diffusion coefficient $D$ is considered to be obtainable by the absorption-desorption experiment when $\alpha \ll 1$ and hence $D=D_{\mathrm{i}}$. Under this condition, the sorption process should be Fickian. In our experimental results, the sorption process for the quenched film is much closer to Fickian than the one for the annealed film. As the result, $\bar{D}_{\mathrm{av}}$ for the quenched film is considered to be not far away in value from the integral diffusion coefficient $\bar{D}$, the integral representation of the mutual diffusion coefficient. On the other hand, $\bar{D}_{\mathrm{av}}^{*}$ for the annealed film is much concerned with the transient sorption process. In other words, in the case of the annealed film, the rate of approach of $D$ to $D_{\mathrm{e}}$ is considered to be the same order as the experimental time scale of the sorption experiment. Therefore the sorption process of the system is clearly non-Fickian. Consequently, the concentration dependence of $\bar{D}_{\mathrm{av}}^{*}$ cannot represent the concentration dependence of the integral diffusion coefficient, the integral representation of the mutual diffusion. coefficient.

The increase in $\alpha$ by annealing can be reasonably explained by assuming that the configurational change of a polymer chain toward its equilibrium, at a fixed concentration, is much retarded by increasing crystallinity because the increase in crystallinity decreases the number of large segments in the amorphous phase, the configuration of which can change quickly.

From the above results, the solubility $S$ and permeability $P$ in the nylon $12+$ water system are proportional to the volume fraction $X_{\mathrm{a}}$ of the amorphous phase, being in contrast with the result obtained by Lasoski, et al. ${ }^{14}$ that $S$ and $P$ for poly(ethylene terephtalate) and nylon 6-10 are proportional to $X_{\mathrm{a}}^{2}$. Our results show that the integral diffusion coefficient $\bar{D}_{\mathrm{s}}$ is independent of crystallinity and hence the mutual diffusion coefficient $D$ is also. In this case the effect of so called "tortusity factor" 15 is considered to be negligible. It is understandable that the diffusion coefficient $D$ in the equilibrium state does not change with crystallinity when we 
consider that the diffusion process takes place only in the amorphous region and that the transfer of a water molecule needs only a local segmental motion of a polymer chain.

Comparison with Other Polymer+Water Systems

In Figure 14, the mutual diffusion coefficient $D$ in the steady state evaluated from the integral diffusion coefficient $\bar{D}_{\mathrm{s}}$ for both the annealed sample and the quenched sample is plotted against $c_{0}{ }^{a}$.

$D_{0}$, the value of $D$ at $c_{0}=0$, for the nylon 12 +water system is plotted against the reciprocal of absolute temperature together with those for other polymer + water systems in Figure 15. The plot for the nylon 12 +water system shows a nonlinear shape which is very similar to that for the nylon $6+$ water system. It is interesting to note that the absolute value of $\log D_{0}$ for nylon $12+$ water system is in between those for hydrophobic polymer (polyethylene and poly(methyl acrylate) [PMA])+water systems and hydrophilic polymer (nylon 6)+water system. This is reasonable, because nylon 12 is more hydrophobic than nylon 6 but more hydrophilic than PMA and polyethylene. It is easy to understand that nylon 12 is more hydrophobic than nylon 6 , considering that nylon 12 has more $\mathrm{CH}_{2}$ groups in a repeating unit than nylon 6 .

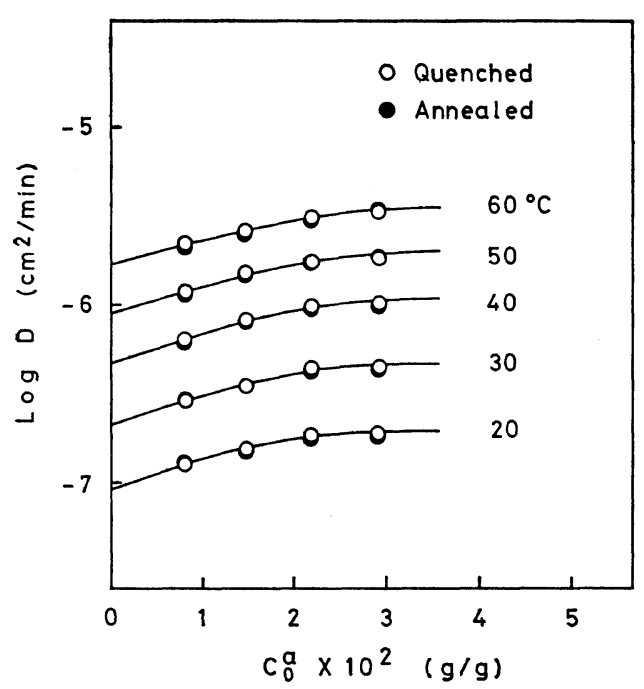

Figure 14. Mutual diffusion coefficient $D$ for nylon $12+$ water system against penetrant concentration $c_{0}{ }^{\mathrm{a}}$.

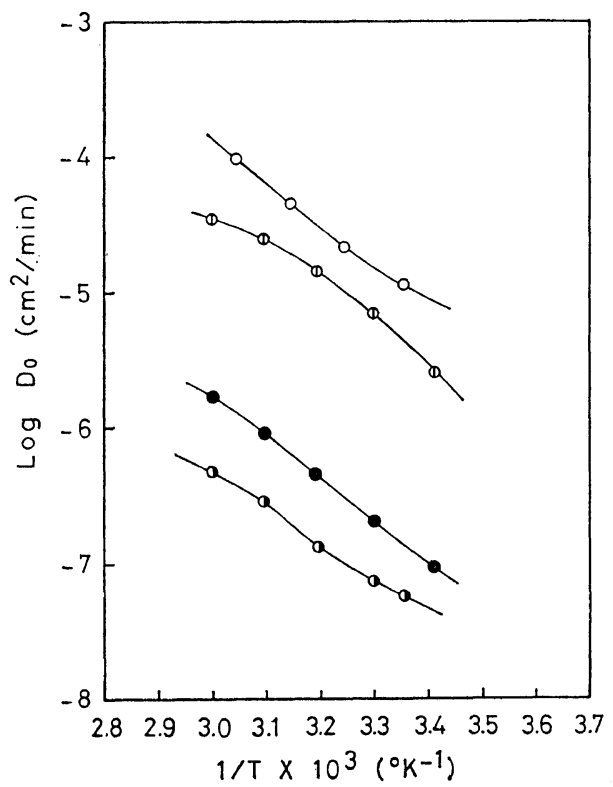

Figure 15. Mutual diffusion coefficient at the limit of zero penetrant concentration as a function of reciprocal absolute temperature: $\bigcirc, \mathrm{PE} ;{ }^{8}(1), \mathrm{PMA}^{7}$ ๑, nylon 12; , nylon $6 .{ }^{2}$

From the slope of the curves in Figure 15 the apparent activation energy $\Delta H_{\mathrm{a}}$ for the diffusion process can be determined. $\Delta H_{\mathrm{a}}$ thus determined for the nylon $12+$ water system is shown in Figure 16 as a function of temperature. The activation energy has a peak at about $40^{\circ} \mathrm{C}$, which corresponds to the glass transition temperature of the sample film in the dry state as

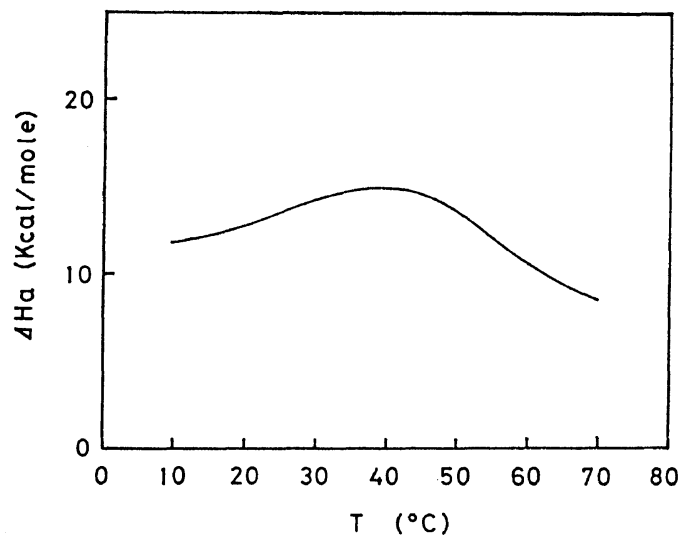

Figure 16. Apparent activation energy for diffusion, $\Delta H_{\mathrm{a}}$, vs. temperature. 
mentioned above. Similar changes of the activation energy with temperature have been observed by us for the nylon $6+$ water system ${ }^{2}$ and Kishimoto, et al., for the poly(vinyl acetate)+ water system. ${ }^{3}$ The decrease in the activation energy above the transition temperature can be explained by an increase in the free volume of the chain molecules in the polymers.

\section{REFERENCES}

1. J. À. Barrie in "Diffusion in Polymers," J. Crank and J.S. Park, Ed., Academic Press, London and New York, 1968, Chapter 8.

2. T. Asada and S. Onogi, J. Colloid Sci., 18, 748 (1963).

3. F. A. Long and L. J. Tompson, J. Polym. Sci., 15, 443 (1955).

4. A. M. Thomas, J. Appl. Chem., 1, 141 (1951).

5. F. Bueche, J. Polym. Sci., 14, 414 (1954).
6. K. Kawasaki and Y. Sekita, J. Appl. Phys. Japan, 26, 678 (1957).

7. A. Kishimoto, E. Maekawa, and H. Fujita, Bull. Chem. Soc. Japan, 33, 988 (1960).

8. H. Yasuda and V. Stannett, J. Polym. Sci., 57, 907 (1962).

9. K. Inoue and S. Hoshino, J. Polym. Sci., Polym. Phys. Ed., 2, 1077 (1973).

10. S. Prager and F. A. Long, J. Amer. Chem. Soc., 73, 4072 (1951).

11. J. Crank, "The Mathematics of Diffusion," Oxford University Press, Oxford, 1956.

12. H. Kurauchi and A. Takeuchi, "Mechanical Behavior of Materials," Vol. 3, Soc. Mater. Sci. Japan, 1972, p 384.

13. J. Crank, J. Polym. Sci., 11, 151 (1953).

14. S. W. Lasoski, Jr. and W. H. Cobbs, Jr., J. Polym. Sci., 36, 31 (1959).

15. A. S. Michaels and R. B. Parker, Jr., ibid., 41, 83 (1959). 